\title{
Inherent irreversibility analysis in a buoyancy induced mag- netohydrodynamic couple stress fluid
}

\author{
Jacob A. Gbadeyann ${ }^{a, b}$, Abiodun A. Opanuga ${ }^{b, *}$ \\ ${ }^{a}$ Department of Mathematics, University of Ilorin, Nigeria. \\ ${ }^{b}$ Department of Mathematics, Covenant University, Nigeria.
}

\begin{abstract}
This paper investigates the inherent irreversibility in a buoyancy induced magnetohydrodynamic (MHD) couple stress fluid through non-Darcian porous medium. It is assumed that the fluid exchanges heat with the ambient following Newtonian law. The governing Navier-Stoke and energy equations are formulated and non-dimensionalied, the approximate solutions for the velocity and temperature profiles are obtained via Adomian decomposition method. The results are used to calculate the entropy generation rate, and Bejan number. The effects of Buoyancy force, suction/injection, Hartman number and other flow parameters on velocity, temperature, entropy generation rate, and Bejan number are analyzed and discussed graphically. The results show that increase in Buoyancy force and suction/injection increases fluid velocity and temperature.Entropy generation rate becomes higher as the values of Buoyancy force, suction/injection parameter, and Hartman number increases.
\end{abstract}

Keywords: Buoyancy force, MHD, porous medium, entropy generation, Adomian decomposition method.

2010 MSC: 76D05, 76W05, 76M25, 34B15.

(C)2018 All rights reserved.

\section{Introduction}

The study of fluid flow and heat transfer in a vertical porous channel have been given considerable attention in the past few decades due to its wide applications in areas such as the design of cooling systems for electronic devices, chemical processing equipment, microelectronic cooling and solar energy, see Jamalabadi et al. [16]. Numerous authors have conducted investigations on such flow; Mutuku-Njane et al. [21] investigated the combined effects of buoyancy force and Navier slip on MHD flow of a Nanofluid over a convectively heated vertical porous plate and submitted that increase in Grashof number decreases the fluid velocity. In the work of Jamalabadi et al. [16], optimal design of Magnetohydrodynamic mixed convection flow in a vertical channel with slip boundary conditions and thermal radiation effects using an entropy generation minimization method was studied. It was concluded that Grashof numbers to Reynolds number ratio are better for maximizing the exergy of the system. Adesanya et al. [4] presented the study of thermodynamic analysis for a third grade fluid through a vertical channel with internal heat

\footnotetext{
*Corresponding author

Email addresses: j.agbadeyan@yahoo.com (Jacob A. Gbadeyan), abiodun.opanuga@covenantuniversity.edu.ng (Abiodun A. Opanuga)
}

doi: $10.22436 /$ jmcs.018.04.03

Received: 2017-05-06 Revised: 2017-06-11 Accepted: 2017-07-29 
generation, among the submissions is that increase in the Grashof number depletes the exergy level of the thermal system. In addition, heat transfer dominates the channel with increase in Grashof number. Also Sharma et al. [29] investigated radiative and free convective effects on MHD flow through a porous medium with periodic wall temperature and heat generation or absorption. It was reported that increase in Grashof number increases the skin-friction coefficient at the wall. Other studies on buoyancy force are found in $[7,15,17,27]$.

Magnetohydrodynamic flow plays a significant role in numerous engineering and industrial processes. Some of these are MHD generators, plasma studies, nuclear reactor, geothermal energy extractions, purifications of metal from non-metal enclosures, polymer technology, and metallurgy, Noor et al. [22]. Excellent researches on MHD flow include Ferdows et al. [14] where the scaling group transformation for MHD boundary layer free convective heat and mass transfer flow past a convectively heated nonlinear radiating stretching sheet was carried out, they observed that increasing magnetic field strength leads to an increase in the shear stress whereas it decreases the rate of heat and mass transfer from the radiate vertical stretching sheet. Sheikholeslami et al. [30] investigated the influence of induced magnetic field on free convection of Nanofluid considering Koo-Kleinstreuer-Li (KKL) correlation, and concluded that velocity profile reduces with rise in the magnetic Prandtl and Hartmann number but it increases with addition of nanoparticles. Adesanya et al. [2] considered the thermodynamics analysis of hydromagnetic third grade fluid flow through a channel filled with porous medium and concluded that increasing magnetic field parameter leads to a reduction in fluid velocity with an increasing effect on fluid temperature. Other related studies on electrically conducting fluid are [11, 18, 19, 31].

Entropy generation analysis is of considerable importance due to its destructive effect. Optimal performance of industrial and engineering flow processes cannot be achieved due to entropy production. It is therefore imperative to analyze the factors responsible for this irreversibility. The current trend of entropy generation analysis is the application of second law of thermodynamics introduced by Bejan [9, 10]. Several other investigations on irreversibility in a system reported in literature under various flow configurations include: Opanuga et al. [25] considered second law analysis of a reactive MHD couple stress fluid through porous channel, the study showed that increase in magnetic field and Frank-Kameneskii parameters increases the entropy generation. Also Opanuga et al. [26] investigated the effect of thermal radiation on the entropy generation of hydromagnetic flow through porous channel, it was stated that entropy generation rises with the increase in the radiation parameter. Adesanya et al. [3] analyzed the effects of couple stresses on entropy generation rate in a porous channel with convective heating. It was reported that increase in couple stress parameter lowers the entropy generation while a rise in Brinkman number increases the entropy generation rate prominently in the middle of the channel than at the walls. Das et al. [12] analyzed entropy generation due to MHD flow in a porous channel with Navier slip. In their report, they stated that entropy generation increases with an increase in magnetic parameter. Other studies on the analysis of entropy generation in various fluid flows can be found in $[1,5,6,8,13,20,28]$.

In this present work, the objective is to analyze the inherent irreversibility in a buoyancy induced MHD couple stress fluid through porous medium. Two semi-analytical techniques, ADM [23] and DTM [24] which have been noted for their high accuracy and rapid convergence are applied. The rest of the work is organized as follows. Section 2 presents the formulation of the problem and non-dimensionalisation. In Section 3 the proposed method of solution to the boundary value problems, Adomian decomposition method is presented. In Section 4 results are graphically discussed while Section 5 contains the concluding remarks.

\section{Model Formulation}

The following assumptions are made:

1. the flow is steady, electrically conducting, and incompressible;

2. the fluid is viscous and flow through vertical porous medium;

3. the channel walls exchange heat with the ambient surrounding following the Newton's cooling law; 
4. a uniform magnetic field of strength $\mathrm{B}_{0}$ is applied;

5. hall effect and induced magnetic field (since magnetic Reynolds number is very small for most fluid used in industrial applications) will be neglected;

6. there is injection at the plate where $y=0$ and suction at the plate where $y=h$ at the same velocity.

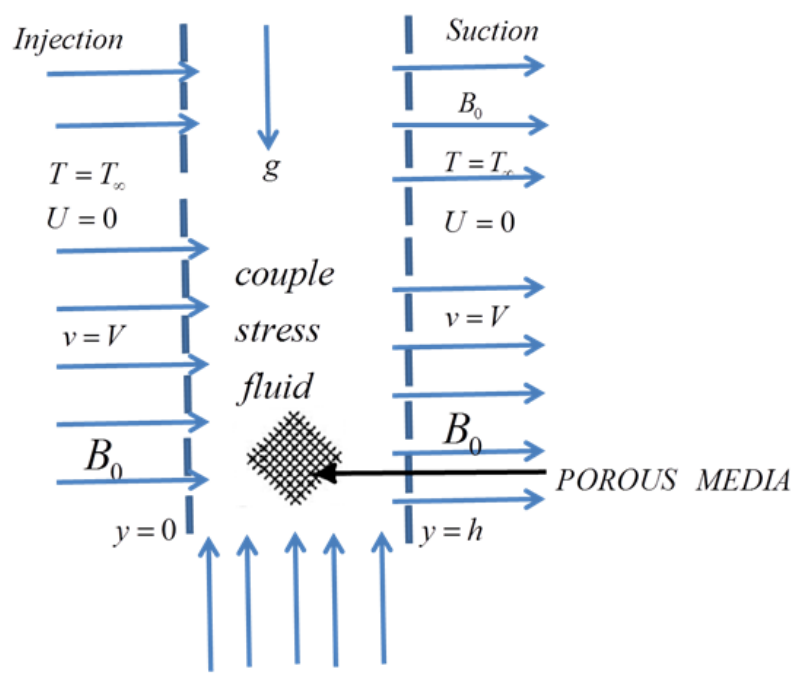

Figure 1: Schematic diagram of the problem.

Then the governing equations for the momentum, heat balance and entropy generation rate can be written as (Adesanya et al. [3])

$$
\begin{aligned}
\rho v_{0} \frac{d u^{\prime}}{d y^{\prime}} & =-\frac{d p}{d x^{\prime}}+\mu \frac{d^{2} u^{\prime}}{d y^{\prime 2}}-\eta \frac{d^{4} u^{\prime}}{d y^{\prime 4}}-\sigma B_{0}^{2} u^{\prime}-\frac{\mu u^{\prime}}{K}-\frac{b u^{\prime 2}}{\sqrt{K}}+g \zeta\left(T-T_{0}\right), \\
\rho c_{p} v_{0} \frac{d T}{d y^{\prime}} & =k \frac{d^{2} T^{\prime}}{d y^{\prime 2}}+\mu\left(\frac{d u^{\prime}}{d y^{\prime}}\right)^{2}+\eta\left(\frac{d^{2} u^{\prime}}{d y^{\prime 2}}\right)^{2}+\sigma B_{0}^{2} u^{\prime 2}+\frac{\mu u^{\prime 2}}{K}+\frac{b u^{\prime 3}}{\sqrt{K}}, \\
E_{G} & =\frac{k}{T_{0}^{2}}\left(\frac{d T^{\prime}}{d y^{\prime}}\right)^{2}+\frac{\mu}{T_{0}}\left(\frac{d u^{\prime}}{d y^{\prime 2}}\right)^{2}+\frac{\eta}{T_{0}}\left(\frac{d^{2} u^{\prime}}{d y^{\prime 2}}\right)^{2}+\frac{\sigma B_{0}^{2} u^{2}}{T_{0}}+\frac{\mu u^{\prime 2}}{T_{0} K}+\frac{b u^{\prime 3}}{T_{0} \sqrt{K}},
\end{aligned}
$$

The boundary conditions are

$$
u^{\prime}(0)=0=\frac{d^{2} u^{\prime}(0)}{d y^{2}}, k \frac{d T^{\prime}(0)}{d y^{\prime}}=-\gamma_{1}\left(T_{f}-T^{\prime}\right), \quad u^{\prime}(h)=0=\frac{d^{2} u^{\prime}(h)}{d y^{2}}, k \frac{d T^{\prime}(h)}{d y^{\prime}}=-\gamma_{2}\left(T^{\prime}-T_{0}\right),
$$

where $u^{\prime}$ is the axial velocity, $\mu$ is the dynamic viscosity, $\rho$ is the fluid density, $T^{\prime}$ is the fluid temperature, $T_{0}$ is the initial fluid temperature, $T_{f}$ is the final fluid temperature, $k$ is the thermal conductivity of the fluid, $c_{p}$ is the specific heat at constant pressure, $v_{0}$ is the constant velocity of fluid suction/injection, $v$ is the kinematic viscosity, $\sigma$ is the electrical conductivity of the fluid, $\eta$ is the fluid particle size effect due to couple stresses, $B_{0}$ is the uniform transverse magnetic field, $\zeta$ is volumetric expansion coefficient and $\mathrm{g}$ is acceleration due to gravity, $E_{\mathrm{G}}$ is the local volumetric entropy generation rate, and $\gamma_{1,2}$ are the heat transfer coefficients.

Now we introduce the following dimensionless variables

$$
\begin{aligned}
& y=\frac{y^{\prime}}{h}, u=\frac{u^{\prime}}{v_{0}}, \theta=\frac{T^{\prime}-T_{0}}{T_{f}-T_{0}}, s=\frac{v_{0} h}{v}, G=-\frac{h^{2} d p}{\mu v_{0} d x}, a^{2}=\mu \frac{h^{2}}{\eta}, \operatorname{Pr}=\frac{v \rho c_{p}}{k}, \\
& B r=\frac{\mu v_{0}^{2}}{k\left(T_{f}-T_{0}\right)}, B i_{1}=\frac{\gamma_{1} h}{k}, B i_{2}=\frac{\gamma_{2} h}{k}, N s=\frac{T_{0}^{2} h^{2} E_{G}}{k\left(T_{f}-T_{0}\right)^{2}}, \Omega=\frac{T_{f}-T_{0}}{T_{0}}, \\
& v=\frac{\mu}{\rho}, H^{2}=\frac{\sigma B_{0}^{2} h^{2}}{\mu}, G r=\frac{g \zeta \rho h^{2}\left(T_{h}-T_{0}\right)}{\mu \nu} .
\end{aligned}
$$

Substituting equation (2.5) into equations (2.1)-(2.4), the following dimensionless equations are obtained 


$$
\begin{aligned}
s \frac{d u}{d y} & =G+\frac{d^{2} u}{d y^{2}}-\frac{1}{a} \frac{d^{4} u}{d y^{4}}-H^{2} u-\beta^{2} u-\alpha^{2} u^{2}+G r \theta \\
\frac{d^{2} \theta}{d y^{2}} & =s \operatorname{Pr} \frac{d \theta}{d y}-B r\left\{\left(\frac{d u}{d y}\right)^{2}+\frac{1}{a^{2}}\left(\frac{d^{2} u}{d y^{2}}\right)^{2}+H^{2} u^{2}+\beta^{2} u^{2}+\alpha^{2} u^{3}\right\}, \\
N s & =\left(\frac{d \theta}{d y}\right)^{2}+\frac{B r}{\Omega}\left\{\left(\frac{d u}{d y}\right)+\frac{1}{a^{2}}\left(\frac{d^{2} u}{d y^{2}}\right)^{2}+H^{2} u^{2}+\beta^{2} u^{2}+\alpha^{2} u^{3}\right\},
\end{aligned}
$$

with the boundary conditions

$$
u(0)=0=\frac{d^{2} u(0)}{d y^{2}}, \frac{d \theta(0)}{d y}=B i_{1}(0)(\theta-1) ; u(1)=0=\frac{d^{2} u(1)}{d y^{2}}, \frac{d \theta(1)}{d y}=-B i_{2}(\theta(1)),
$$

where $u$ is the dimensionless velocity, $s$ is the suction/injection parameter, $\theta$ is the dimensionless temperature, $\mathrm{a}$ is the couple stress parameter, $\mathrm{Pr}$ is the Prandtl number, $\mathrm{Br}$ is the Brinkman number, $\Omega$ is the parameter that measures the temperature difference between the two heat reservoirs, $\mathrm{H}^{2}$ is the magnetic field parameter, $\mathrm{Ns}$ is the dimensionless entropy generation rate, $\mathrm{Be}$ and $\mathrm{Bi}_{1,2}$ are the Bejan number and Biot numbers respectively, $\mathrm{G}$ is the axial pressure gradient and $\mathrm{Gr}$ is the Grashof number.

\section{Method of solution}

The integral form of equations (2.6) and (2.7) are of the form

$$
u(y)=a_{1} y+\frac{a_{2}}{3 !} y^{3}+a^{2} \int_{0}^{y} \int_{0}^{y} \int_{0}^{y} \int_{0}^{y}\left\{G+\frac{d^{2} u}{d Y^{2}}-s \frac{d u}{d Y}-H^{2} u-\beta^{2} u-\alpha^{2} u^{2}+G r \theta\right\} d Y d Y d Y d Y,
$$

and

$$
\theta(y)=a_{3}+a_{4}(y)+\int_{0}^{y} \int_{0}^{y}\left\{\operatorname{sPr} \frac{d \theta}{d Y}-\operatorname{Br}\left\{\left(\frac{d u}{d Y}\right)^{2}+\frac{1}{a^{2}}\left(\frac{d^{2} u}{d Y^{2}}\right)^{2}+H^{2} u^{2}+\beta^{2} u^{2}+\alpha^{2} u^{3}\right\}\right\} d Y d Y,
$$

where $a_{1}, a_{2}, a_{3}, a_{4}$ are the parameters to be determined later. By ADM, we define an infinite series solution of the form

$$
u(y)=\sum_{n=0}^{\infty} u_{n}(y), \quad \theta(y)=\sum_{n=0}^{\infty} \theta_{n}(y) .
$$

Now using (3.3) in (3.1)-(3.2) yields

$$
\begin{aligned}
\sum_{n=0}^{\infty} u_{n}(y)= & a_{1} y+\frac{a_{2}}{3 !} y^{3}+a^{2} \int_{0}^{y} \int_{0}^{y} \int_{0}^{y} \int_{0}^{y}\left\{G+\sum_{n=0}^{\infty} \frac{d^{2} u_{n}}{d Y^{2}}-s \sum_{n=0}^{\infty} \frac{d u_{n}}{d Y}\right. \\
& \left.-H^{2} \sum_{n=0}^{\infty} u_{n}-\beta^{2} \sum_{n=0}^{\infty} u_{n}-\alpha^{2} \sum_{n=0}^{\infty} u_{n}^{2}+G r \sum_{n=0}^{\infty} \theta_{n}\right\} d Y d Y d Y d Y
\end{aligned}
$$

and

$$
\begin{aligned}
\sum_{n=0}^{\infty} \theta_{n}(y)= & a_{3}+a_{4} y+\int_{0}^{y} \int_{0}^{y}\left\{s \operatorname{Pr} \sum_{n=0}^{\infty} \frac{d \theta_{n}}{d Y}-B r\left\{\left(\sum_{n=0}^{\infty} \frac{d u_{n}}{d Y}\right)^{2}+\frac{1}{a^{2}}\left(\sum_{n=0}^{\infty} \frac{d^{2} u_{n}}{d Y^{2}}\right)^{2}\right.\right. \\
& \left.\left.+H^{2} \sum_{n=0}^{\infty} u_{n}^{2}+\beta^{2} \sum_{n=0}^{\infty} u_{n}^{2}+\alpha^{2} \sum_{n=0}^{\infty} u_{n}^{3}\right\}\right\} d Y d Y .
\end{aligned}
$$

From equation (3.4)-(3.5), the zeroth order term can be written as

$$
\sum_{n=0}^{\infty} u_{0}(y)=a_{1} y+\frac{a_{2}}{3 !} y^{3}+a^{2} \int_{0}^{y} \int_{0}^{y} \int_{0}^{y} \int_{0}^{y}\{G\}
$$




$$
\sum_{n=0}^{\infty} \theta_{0}(y)=a_{3}+a_{4} y
$$

while other terms can be determined using the recurrence relations

$$
\begin{aligned}
\sum_{n=0}^{\infty} u_{n+1}(y)= & a^{2} \int_{0}^{y} \int_{0}^{y} \int_{0}^{y} \int_{0}^{y}\left\{\sum_{n=0}^{\infty} \frac{d^{2} u_{n}}{d Y^{2}}-s \sum_{n=0}^{\infty} \frac{d u_{n}}{d Y}-H^{2} \sum_{n=0}^{\infty} u_{n}\right. \\
& \left.-\beta^{2} \sum_{n=0}^{\infty} u_{n}-\alpha^{2} \sum_{n=0}^{\infty} u_{n}^{2}+G r \sum_{n=0}^{\infty} \theta_{n}\right\} d Y d Y d Y d Y,
\end{aligned}
$$

and

$$
\begin{aligned}
\sum_{n=0}^{\infty} \theta_{n+1}(y)= & \int_{0}^{y} \int_{0}^{y}\left\{s \operatorname{Pr} \sum_{n=0}^{\infty} \frac{d \theta_{n}}{d Y}-B r\left\{\left(\sum_{n=0}^{\infty} \frac{d u_{n}}{d Y}\right)^{2}+\frac{1}{a^{2}}\left(\sum_{n=0}^{\infty} \frac{d^{2} u_{n}}{d Y^{2}}\right)^{2}\right.\right. \\
& \left.\left.+H^{2} \sum_{n=0}^{\infty} u_{n}^{2}+\beta^{2} \sum_{n=0}^{\infty} u_{n}^{2}+\alpha^{2} \sum_{n=0}^{\infty} u_{n}^{3}\right\}\right\} d Y d Y .
\end{aligned}
$$

The nonlinear terms in equation (3.9) are written as

$$
B_{n}=u^{2}, C_{n}=u^{3},
$$

while the Adomian polynomials are computed as

$$
B_{0}=u_{0}^{2}, B_{1}=2 u_{0} u_{1}, B_{2}=2 u_{0} u_{2}+u_{1}^{2} ; C_{0}=u_{0}^{3}, C_{1}=3 u_{0}^{2} u_{1}, C_{2}=3 u_{0}^{2} u_{2}+3 u_{0} u_{1}^{2} .
$$

Substituting equation (3.10) in (3.9) yields

$$
\begin{aligned}
\sum_{n=0}^{\infty} \theta_{n+1}(y)= & \int_{0}^{y} \int_{0}^{y}\left\{s \operatorname{sr} \sum_{n=0}^{\infty} \frac{d \theta_{n}}{d Y}-B r\left\{\left(\sum_{n=0}^{\infty} \frac{d u_{n}}{d Y}\right)^{2}+\frac{1}{a^{2}}\left(\sum_{n=0}^{\infty} \frac{d^{2} u_{n}}{d Y^{2}}\right)^{2}\right.\right. \\
& \left.\left.H^{2} \sum_{n=0}^{\infty} B_{n}+\beta^{2} \sum_{n=0}^{\infty} B_{n}+\alpha^{2} \sum_{n=0}^{\infty} C_{n}\right\}\right\} d Y d Y .
\end{aligned}
$$

Solving equations (3.6)-(3.8) and (3.11) at $n=0,1,2,3, \ldots$ gives the series solution of the boundary value problems.

Table 1: Computation showing convergence of solution for $s=0.1, \mathrm{H}=\mathrm{a}=\mathrm{Br}=\mathrm{Bi} i_{1}=\mathrm{Bi}_{2}=\mathrm{G}=1, \beta=0.01, \alpha=0, \mathrm{Gr}=0$.

\begin{tabular}{|c|c|c|c|c|c|}
\hline $\mathbf{y}$ & Exact solution & $\mathrm{U}_{\mathrm{DTM}}$ & $\mathrm{U}_{\mathrm{ADM}}$ & ADM Abs Error & DTM Abs Error \\
\hline 0 & $5.55 \times 10^{-17}$ & 0.000000000 & 0.000000000 & $5.55 \times 10^{-17}$ & $5.55 \times 10^{-17}$ \\
\hline 0.1 & 0.003679982 & 0.003679982 & 0.003679982 & $1.88 \times 10^{-12}$ & $1.08 \times 10^{-11}$ \\
\hline 0.2 & 0.006958985 & 0.006958985 & 0.006958985 & $3.72 \times 10^{-12}$ & $2.13 \times 10^{-11}$ \\
\hline 0.3 & 0.009523084 & 0.009523084 & 0.009523084 & $5.47 \times 10^{-12}$ & $3.11 \times 10^{-11}$ \\
\hline 0.4 & 0.011150296 & 0.011150296 & 0.011150296 & $7.12 \times 10^{-12}$ & $3.99 \times 10^{-11}$ \\
\hline 0.5 & 0.011708189 & 0.011708189 & 0.011708189 & $8.66 \times 10^{-12}$ & $4.73 \times 10^{-11}$ \\
\hline 0.6 & 0.011152495 & 0.011152495 & 0.011152495 & $1.01 \times 10^{-11}$ & $5.30 \times 10^{-11}$ \\
\hline 0.7 & 0.009526717 & 0.009526717 & 0.009526717 & $1.15 \times 10^{-11}$ & $5.66 \times 10^{-11}$ \\
\hline 0.8 & 0.006962734 & 0.006962734 & 0.006962734 & $1.28 \times 10^{-11}$ & $5.79 \times 10^{-11}$ \\
\hline 0.9 & 0.003682379 & 0.003682379 & 0.003682379 & $1.42 \times 10^{-11}$ & $5.63 \times 10^{-11}$ \\
\hline
\end{tabular}

All the computations of the integral equations (3.6)-(3.8) and (3.11) are done by coding the equations in an algebra symbolic package-Mathematica. Only graphical results are presented in Figures 2-13 due to the large size of the results. The accuracy of the result obtained via ADM is validated using the DTM and the exact solution. The comparison is presented in Table 1. In the result section, the results obtained for the velocity and temperature fields are used to compute the entropy generation rate. 


\subsection{Entropy Generation}

The expression for entropy generation Bejan [11] in equation (2.3) given below suggests five sources of entropy production. The first term is irreversibility due to heat transfer, the second term is irreversibility due to fluid friction while the last three terms are entropy generation due to couple stresses, magnetic field and porosity respectively.

$$
E_{G}=\frac{k}{T_{0}^{2}}\left(\frac{d T^{\prime}}{d y^{\prime}}\right)^{2}+\frac{\mu}{T_{0}}\left(\frac{d u^{\prime 2}}{d y^{\prime}}\right)^{2}+\frac{\eta}{T_{0}}\left(\frac{d^{2} u^{\prime}}{d y^{\prime 2}}\right)^{2}+\frac{\sigma B_{0}^{2} u^{2}}{T_{0}}+\frac{\mu u^{\prime 2}}{T_{0} K}+\frac{b u^{\prime 3}}{T_{0} \sqrt{K}} .
$$

The dimensionless form in equation (2.8) is also written as

$$
N s=\left(\frac{d \theta}{d y}\right)^{2}+\frac{B r}{\Omega}\left\{\left(\frac{d u}{d y}\right)^{2}+\frac{1}{a^{2}}\left(\frac{d^{2} u}{d y^{2}}\right)^{2}+H^{2} u^{2}+\beta^{2} u^{2}+\alpha^{2} u^{3}\right\},
$$

entropy generation rate is investigated by setting

$$
N_{1}=\left(\frac{d \theta}{d y}\right)^{2}, N_{2}=\frac{B r}{\Omega}\left\{\left(\frac{d u}{d y}\right)^{2}+\frac{1}{a^{2}}\left(\frac{d^{2} u}{d y^{2}}\right)^{2}+H^{2} u^{2}+\beta^{2} u^{2}+\alpha^{2} u^{3}\right\}
$$

where $N_{1}$ is irreversibility due to heat transfer and $N_{2}$ represents fluid friction irreversibility with couple stresses, magnetic field and porosity. To describe the contribution of heat transfer irreversibility to the overall entropy generation the Bejan number Be is employed, it gives the ratio of heat transfer and viscous dissipation with magnetic field within the channel as

$$
\mathrm{Be}=\frac{\mathrm{N}_{1}}{\mathrm{~N}_{\mathrm{s}}}=\frac{1}{1+\Phi^{\prime}}
$$

where $\Phi=\frac{N_{2}}{N_{1}}$ is the irreversibility ratio. Equation (3.12) indicates that Bejan number takes values between 0 and 1, i.e., $0 \leqslant \mathrm{~B} e \leqslant 1$. The value $\mathrm{Be}=1$ signifies the limit at which heat transfer dominates entropy generation, $\mathrm{Be}=0$ gives the limit at which viscous dissipation and magnetic field dominate while $\mathrm{Be}=0.5$ represents equal contribution of both heat transfer and viscous dissipation to entropy production.

\section{Results and discussion}

In this present study, inherent irreversibility in a buoyancy induced MHD couple stress fluid through porous medium has been investigated using the rapidly convergent Adomian decomposition method. The velocity and temperature profiles are obtained and used to compute the entropy generation rate. The effects of various parameters are considered on velocity, temperature, entropy generation, and Bejan number to provide insight to the problems, and the results are graphically displayed in Figs. 2-13.

\subsection{Effect of parameters variation on velocity profile}

Figs. 2-4 present the variations of velocity for different flow parameters. Fig. 2 represents the effect of variation in Grashof number on velocity profile. There is a rise in the velocity of the fluid as Grashof number increases. It is observed that buoyancy force speeds up fluid velocity. Fig. 3 presents the effect of suction/injection on fluid velocity. The plot shows a rise in fluid velocity as suction/injection parameter increases, the rise in fluid velocity is due to the reduction in fluid kinematic viscosity as more hot fluid is injected into the channel. In Fig. 4 the effect of magnetic field parameter on fluid velocity is depicted, it is observed that an increase in the magnetic field parameter retards fluid velocity. The is caused by the applied magnetic field which clusters fluid particles and thus impedes the free flow of the fluid. 


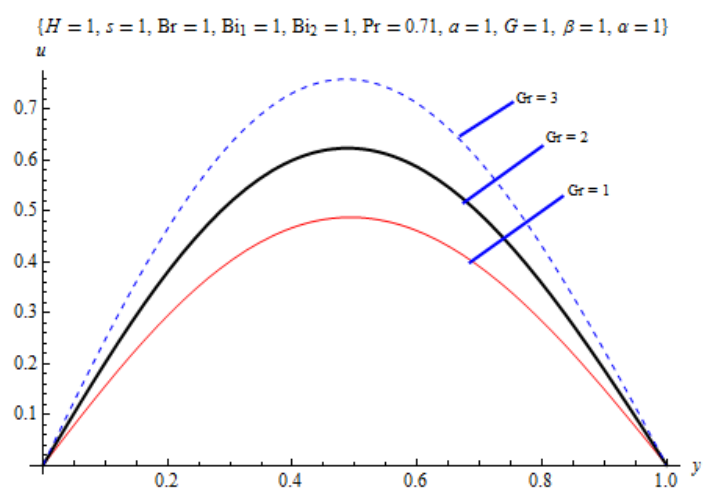

Figure 2: Effect of Grashof number (Gr) on velocity profile.

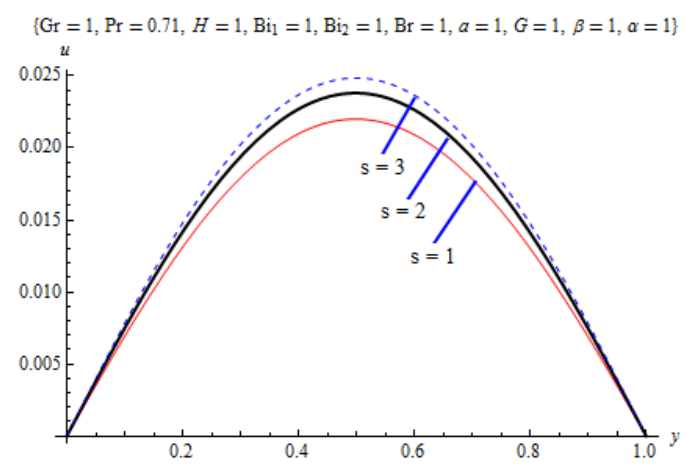

Figure 3: Effect of suction/injection (s) on velocity profile.

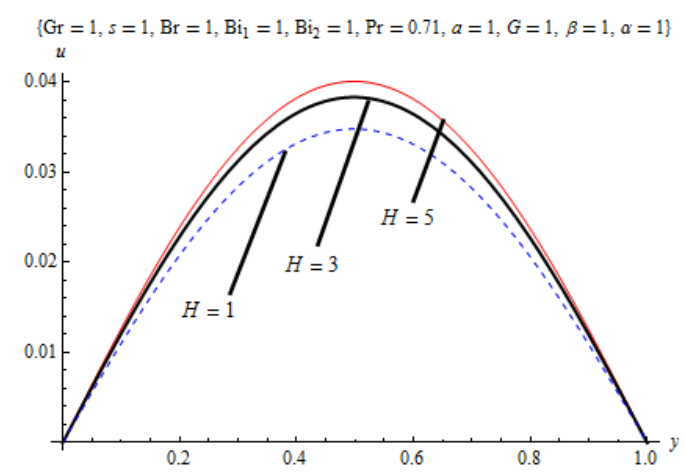

Figure 4: Effect of magnetic field $\left(\mathrm{H}^{2}\right)$ on velocity profile.

\subsection{Effect of parameters variation on temperature profile}

The influence of different governing parameters on temperature are presented in Figs. 5-7. Fig. 5 portrays the effect of Grashof number on fluid temperature. Fluid temperature is enhanced across the channel as the value of Grashof number increases due to increase in fluid volumetric expansion. Fig. 6 displays the effect of suction/injection parameter on fluid temperature; the Figure reveals that temperature increases as suction/injection parameter increases. This is true since injection of hot fluid into the channel will definitely increase fluid temperature. The effect of magnetic field parameter on fluid temperature is shown in Fig. 7. It is clear from the plot that fluid temperature increases as magnetic field parameter increases. The Lorentz heating effect is attributed to the rise in temperature. 


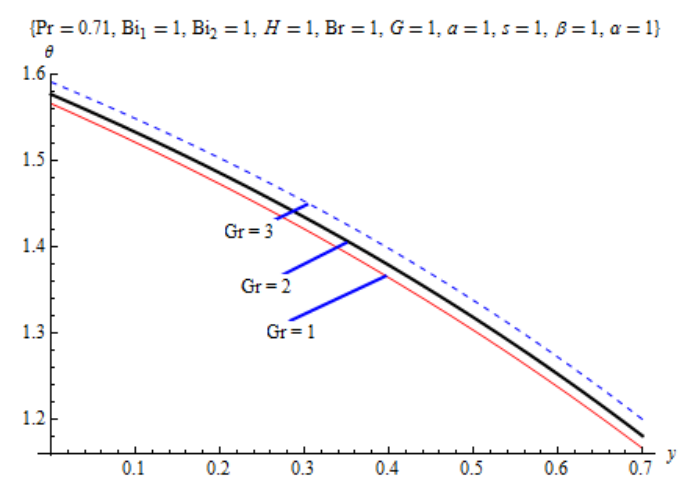

Figure 5: Effect of Grashof number (Gr) on temperature profile.

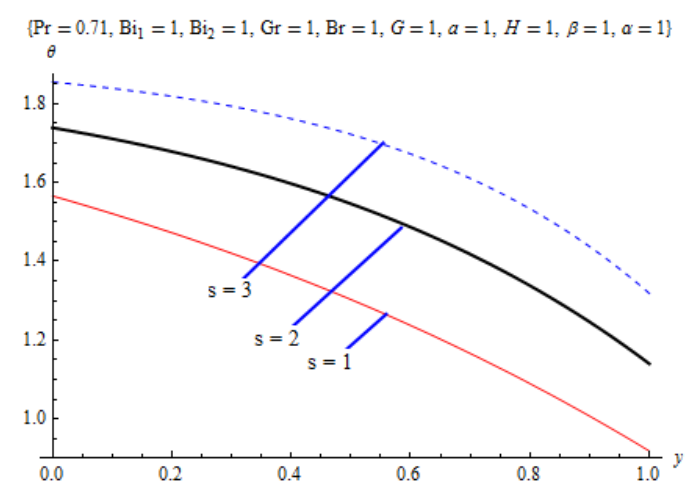

Figure 6: Effect of suction/injection (s) on temperature profile.

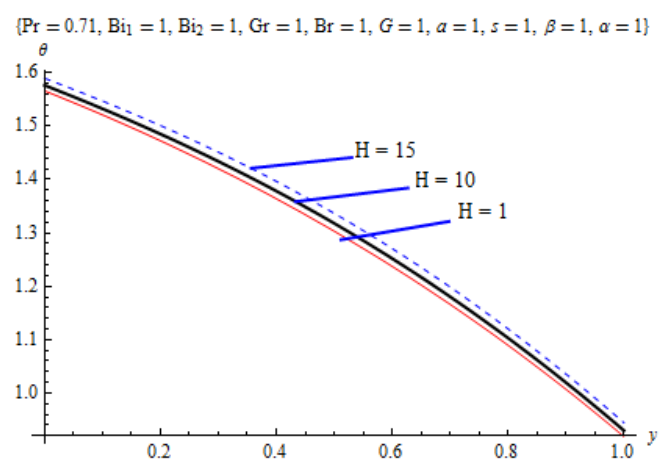

Figure 7: Effect of magnetic field parameter $\left(\mathrm{H}^{2}\right)$ on temperature profile.

\subsection{Effect of parameters variation on entropy generation rate}

The influences of various parameters on Entropy generation rate are presented in Figs. 8-10. Fig. 8 describes the effect of Grashof number on entropy generation rate. The plot depicts that entropy generation registers an increase as Grashof number rises in values. Recall that in Figs. 2 and 5, it is presented that fluid velocity and temperature increased as Grashof number increases due to the impact of buoyancy force as well as rise in fluid volumetric expansion respectively. The net effect is the increased randomness of fluid particles which results in increased entropy production. Furthermore Fig. 9 depicts the influence of suction/injection on entropy generation rate, from the plot it is observed that entropy generation increases considerably as suction/injection parameter varies. This is because of the injection of hot fluid into the channel which resulted in increased velocity and temperature as revealed in Figs. 3 and 6.

Figure 10 displays the effect of magnetic field parameter on entropy generation. Entropy production 
becomes higher at the upper wall as magnetic field parameter rises. This is explained in Fig. 7 that increase in Hartman number has a corresponding increase in fluid temperature because the applied magnetic field clustered fluid particles together thereby enhancing viscous dissipation. Consequently, fluid velocity is reduced and the temperature rises leading to a rise in entropy generation.

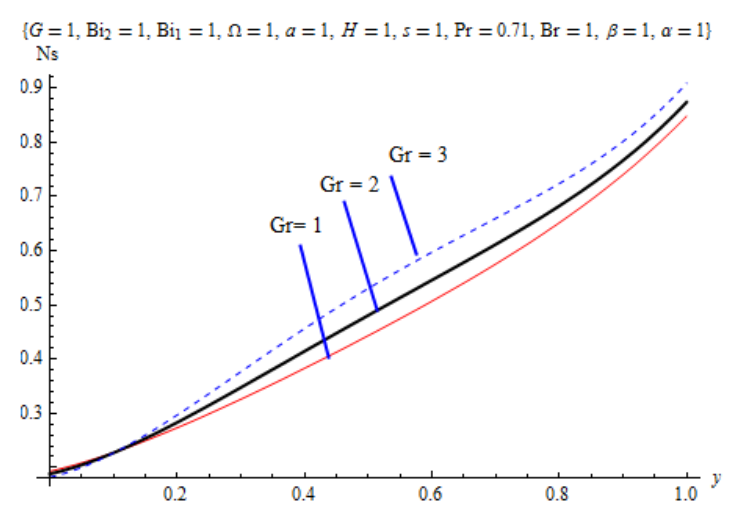

Figure 8: Effect of Grashof number (Gr) on entropy generation rate.

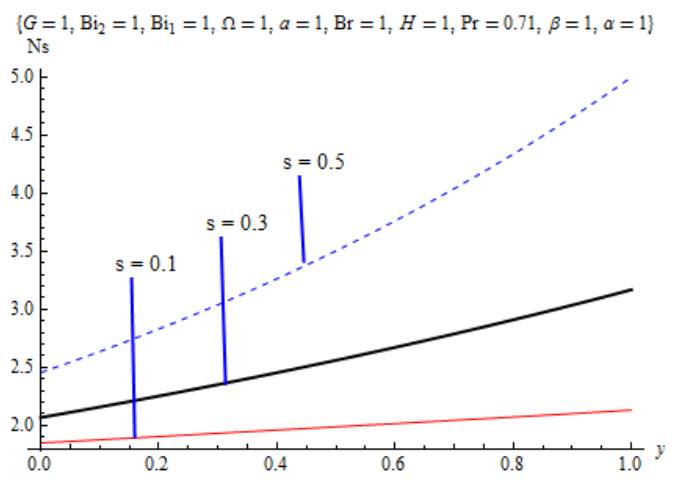

Figure 9: Effect of suction/injection (s) on entropy generation rate.

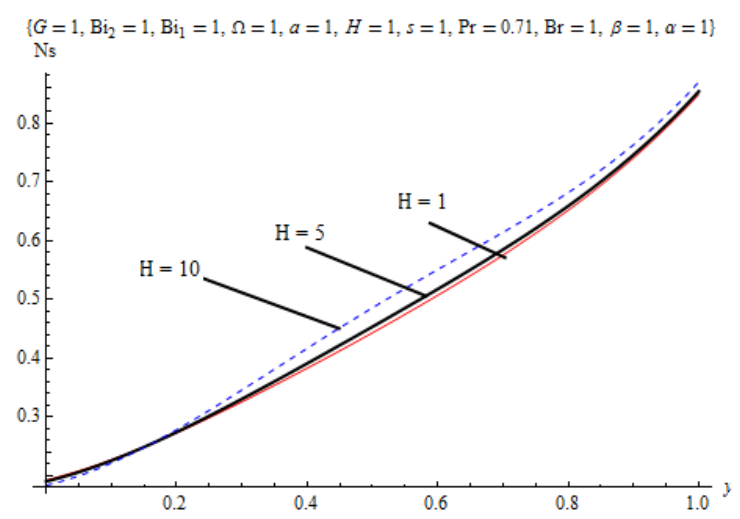

Figure 10: Effect of magnetic field parameter $\left(\mathrm{H}^{2}\right)$ on entropy generation rate.

\subsection{Effect of parameters variation on Bejan number}

This section presents the effect of variation in parameters on Bejan number in Figs. 11-13. Fig. 11 depicts the effect of Grashof number on Bejan number, the plots indicate that Bejan number decreases 
as the values of Grashof number increases. Therefore entropy generation due to viscous dissipation dominates over heat transfer. Figs. 12 and 13 display the plots of Hartman number and suction/injection parameters respectively on Bejan number, the plots show that Bejan number increases as the values of the parameters increase. This is an indication that heat transfer dominates entropy generation for Hartman number and suction/injection.

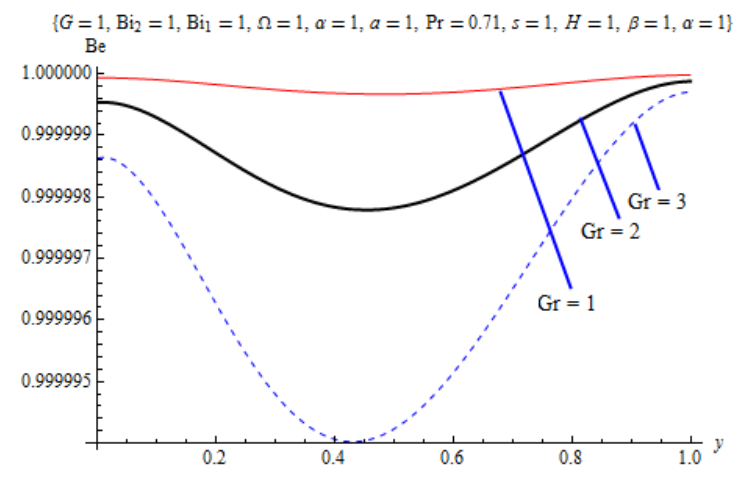

Figure 11: Effect of Grashof number (Gr) on Bejan number.

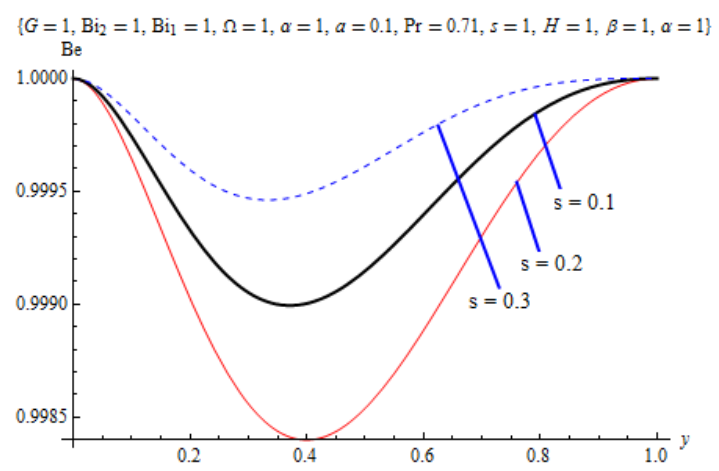

Figure 12: Effect of suction/injection (s) on Bejan number.

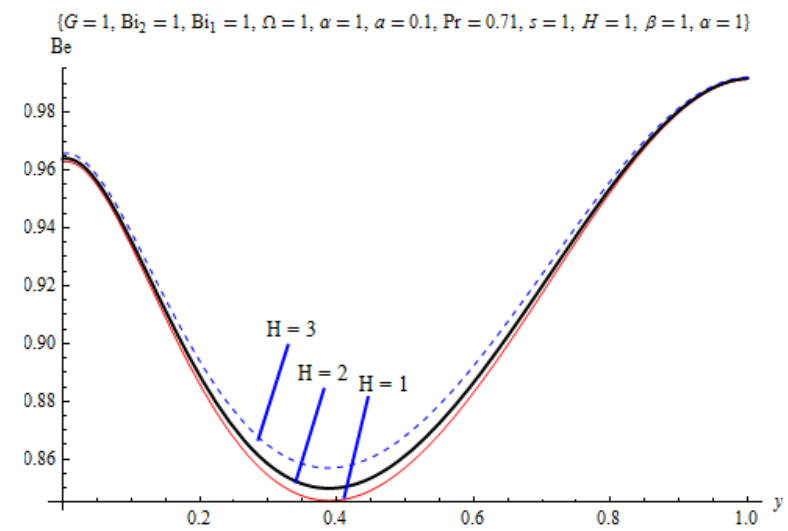

Figure 13: Effect of magnetic field parameter $\left(\mathrm{H}^{2}\right)$ on Bejan number. 


\section{Conclusions}

In this study inherent irreversibility analysis in a buoyancy induced MHD couple stress fluid has been analyzed, the study shows among other things that:

i increase in buoyancy force and suction/injection parameter increases fluid velocity;

ii increase in buoyancy force, magnetic field, and suction/injection parameters raises fluid temperature;

iii entropy generation rate becomes higher as the values of Grashof number, suction/injection parameter, and Hartman number increases;

iv increase in buoyancy force reduces Bejan number while suction/injection parameter and Hartman number increases Bejan number indicating that both heat transfer and viscous dissipation contribute to entropy production.

\section{Acknowledgment}

The authors would like to appreciate Covenant University, Ota, Nigeria for financial support and the anonymous reviewers for their comments.

\section{References}

[1] S. O. Adesanya, Second law analysis for third-grade fluid with variable properties, J. Therm., 2014 (2014), 8 pages. 1

[2] S. O. Adesanya, J. A. Falade, Thermodynamics analysis of hydromagnetic third grade fluid flow through a channel filled with porous medium, Alexandria Eng. J., 54 (2015), 615-622. 1

[3] S. O. Adesanya, O. D. Makinde, Effects of couple stress on entropy generation rate in a porous channel with convective heating, Comput. Appl. Math., 34 (2015), 293-307. 1, 2

[4] S. O. Adesanya, O. D. Makinde, Thermodynamic analysis for a third grade fluid through a vertical channel with internal heat generation, J. Hydrodynamics Ser. B, 27 (2015), 264-272. 1

[5] A. O. Ajibade, B. K. Jha, A. Omame, Entropy generation under the effect of suction/injection, Appl. Math. Model., 35 (2011), 4630-4646. 1

[6] A. Arikoglu, I. Ozkol, G. Komurgoz, Effect of slip on entropy generation in a single rotating disk in MHD flow, Appl. Energy, 85 (2008), 1225-1236. 1

[7] A. Barletta, Laminar convection in a vertical channel with viscous dissipation and buoyancy effects, Int. Commun. Heat Mass, 26 (1999), 153-164. 1

[8] T. Basak, R. S. Kaluri, A. R. Balakrishnan, Entropy generation during natural convection in a porous cavity: Effect of thermal boundary conditions, Numer. Heat Transfer Part A: Appl., 62 (2012), 336-364. 1

[9] A. Bejan, Second-law analysis in heat transfer and thermal design, Adv. Heat Transfer, 15 (1982), 1-58. 1

[10] A. Bejan, Second law analysis in heat transfer, Energy int. J., 15 (1980), 721-732. 1

[11] M. M. Bhatti, A. Zeeshanb, R. Ellahi, N. Ijaz, Heat and mass transfer of two-phase flow with Electric double layer effects induced due to peristaltic propulsion in the presence of transverse magnetic field, J. Mol. Liq., 230 (2017), 237-246. 1, 3.1

[12] S. Das, R. N. Jana, Entropy generation due to MHD flow in a porous channel with Navier slip, Ain Shams Eng. J., 5 (2014), 575-584. 1

[13] A. El Jery, N. Hidouri, M. Magherbi, A. B. Brahim, Effect of an External Oriented Magnetic Field on Entropy Generation in Natural Convection, Entropy, 12 (2010), 1391-1417. 1

[14] M. Ferdows, M. J. Uddin, A. A. Afify, Scaling group transformation for MHD boundary layer free convective heat and mass transfer flow past a convectively heated nonlinear radiating stretching sheet, Int. J. Heat Mass Transfer, 56 (2013), 181-187. 1

[15] O. M. Hadaad, M. M. Abuzaid, M. A. Al-Nimr, Developing free-convection gas flow in vertical open-ended michrochannel filled with porous media, Int. J. comput., 48 (2005), 693-710. 1

[16] M. Y. A. Jamalabadi, J. H. Park, C. Y. Lee, Optimal design of magnetohydrodynamic mixed convection flow in a vertical channel with slip boundary conditions and thermal radiation effects by using an entropy generation minimization method, Entropy, 17 (2015), 866-881. 1

[17] B. K. Jha, A. O. Ajibade, Free convective flow of heat generation absorbing fluid between vertical porous plates with periodic heat input, Int. J. Heat Mass Transfer, 36 (2009), 624-631. 1

[18] A. A. Khan, S. Muhammad, R. Ellahi, Q. M. Zaigham Zia, Bionic Study of Variable Viscosity on MHD Peristaltic Flow of Pseudoplastic Fluid in an Asymmetric Channel, J. Magn., 21 (2016), 273-280. 1

[19] A. A. Khana, H. Usmana, K. Vafai, R. Ellahi, Study of peristaltic flow of magnetohydrodynamic Walter's B fluid with slip and heat transfer, Scientia Iranica, 23 (2016), 2650-2662. 1 
[20] A. Mazgar, F. Ben Nejma, K. Charrada, Entropy generation through combined non-grey gas radiation and forced convection in cylinders, 6th IASME/WSEAS International Conference on heat transfer, thermal engineering and environment (Rhodes, Greece), 2008 (2008), 11 pages. 1

[21] W. N. Mutuku-Njane, O. D. Makinde, Combined effects of buoyancy force and Navier slip on MHD flow of a Nanofluid over a convectively heated vertical porous plate, The Scientific World Journal, 2013 (2013), 8 pages. 1

[22] N. F. M. Noor, S. Abbasbandy, I. Hashim, Heat and mass transfer of thermophoretic MHD flow over an inclined radiate isothermal permeable surface in the presence of heat source/sink, Int. J. Heat Mass Transfer, 55 (2012), 2122-2128. 1

[23] A. A. Opanuga, O. O. Agboola, H. I. Okagbue, Approximate solution of multipoint boundary value problems, J. Eng. Appl. Sci., 10 (2015), 85-89. 1

[24] A. A. Opanuga, O. O. Agboola, H. I. Okagbue, J. G. Oghonyon, Solution of differential equations by three semianalytical techniques, Int. J. Appl. Eng. Res., 10 (2015), 39168-39174. 1

[25] A. A. Opanuga, J. A. Gbadeyan, S. A. Iyase, Second law analysis of a reactive MHD couple stress fluid through porous channel, Int. J. Appl. Math. Statist., 56 (2017), 85-100. 1

[26] A. A. Opanuga, J. A. Gbadeyan, S. A. Iyase, H. I. Okagbue, Effect of thermal radiation on entropy generation of hydromagnetic flow through porous channel, Pacific J. Sci. Tech., 17 (2016), 59-68. 1

[27] I. Ozkol, G. Komurgoz, A. Arikoglu, Entropy generation in laminar natural convection from a constant temperature vertical plate in an infinite fluid, Proceedings of the institution of mechanical engineers part A: J. Power Energy, 221 (2007), 609-616. 1

[28] M. Pakdemirli, B. S. Yilbas, Entropy generation in a pipe due to non-Newtonian fluid flow: Constant viscosity case, Sadhana, 31 (2006), 21-29. 1

[29] P. R. Sharma, K. Sharma, T. Mehta, Radiative and free convective effects on MHD flow through a porous medium with periodic wall temperature and heat generation or absorption, Int. J. Math. Arch., 5 (2014), 119-128. 1

[30] M. Sheikholeslami, Q. M. Zaigham Zia, R. Ellahi, Influence of Induced Magnetic Field on Free Convection of Nanofluid Considering Koo-Kleinstreuer-Li (KKL) Correlation, Appl. Sci., 6 (2016), 1-11. 1

[31] A. Zeeshan, A. Majeed, R. Ellahi, Effect of magnetic dipole on viscous ferro-fluid past a stretching surface with thermal radiation, J. Mol. Liq., 215 (2016), 549-554. 1 